REVIEW

\title{
Genetic pathways to melanoma tumorigenesis
}

\section{R Hussein}

J Clin Pathol 2004;57:797-801. doi: 10.1136/icp.2003.015800

The incidence of cutaneous malignant melanomas is growing faster than that of any other cancer and therefore posing a major heath threat worldwide. In melanocytic skin tumours, the feasibility of correlating a specific pathological stage with a corresponding genetic alteration provides a remarkable opportunity to study the multistep tumorigenesis model. This multistep melanoma tumorigenesis is best described as a continuum of transformation of the melanocytes, melanocytic dysplasia, and melanoma formation. These steps involve genotypic alterations including loss of tumour suppressor genes, microsatellite instability, and alterations of the mismatch repair system. This review seeks to examine melanoma tumorigenesis based on these genetic changes.

$\mathrm{T}$ he melanocytic system encompasses a full range of lesional steps from benign naevi to metastatic malignancy. Because melanocytes have the unique capability to produce melanin, their proliferation is readily apparent. This unique feature, combined with the ability to observe these lesions, has enabled six lesional steps to be recorded during melanoma tumorigenesis, namely: (1) common acquired melanocytic naevus (BN); (2) melanocytic naevus with lentigenious melanocytic hyperplasia-that is, aberrant differentiation; (3) melanocytic naevus with aberrant differentiation and melanocytic nuclear atypia-that is, melanocytic dysplasia; (4) radial growth phase of primary melanoma (RGP-CMM); (5) vertical growth phase of primary melanoma (VGP-CMM); and (6) metastatic melanoma. ${ }^{1}$ BN represents the initial step in the course of melanocytic proliferation. Naevus cells derived from melanocytes among the basal cells proliferate along the dermoepidermal junction to establish a junctional melanocytic hyperplasia-that is, lentigenious melanocytic hyperplasia. At this stage, one group of these lesions remains stable-naevi with lesional persistence and stability. The second group continues to grow and follows either the pathway of differentiation or that of aberrant differentiation and melanocytic nuclear atypiamelanocytic dysplasia. In RGP-CMM, there is a sequential focal progression of the genetically initiated melanocytic dysplastic naevi (MDN) to RGP-CMM. This progression represents the first stage of cancer in the melanocytic system. This transition is of particular importance because the growth of the preceding lesional steps ( $\mathrm{BN}$ and $\mathrm{MDN}$ ) is limited and self-controlled. In contrast, RGP-CMM cells have the ability to grow both
Correspondence to: Assiut University Hospitals, Assiut, Egypt; mrh17@ swissnfo.org indefinitely and independently; that is, they have partial growth autonomy. Once this autonomy is acquired, RGP-CMM develops into metastatic melanoma. ${ }^{2}$ The subsequent progression of RGPCMM to VGP-CMM is the most crucial step in melanoma tumorigenesis. During this transition, RGP-CMM cells acquire more growth autonomy and proceed to VGP-CMM. Therefore, this stage signals the potential for further progression being the rule rather than the exception. ${ }^{3}$

"Radial growth phase of primary melanoma cells have the ability to grow both indefinitely and independently; that is, they have partial growth autonomy"

Multistep tumorigenesis is a process that involves inactivation of tumour suppressor genes (TSGs), activation of oncogenes, and defects in housekeeping genes such as mismatch repair (MMR) genes. ${ }^{4}$ This model can be easily adapted to CMMs, because they have fairly well characterised clinical precursors such as melanocytic dysplastic naevi (MDN) and an early nontumorigenic phase-RGP-CMM. From a histopathological prospective, CMMs develop from a transformed melanocyte by a series of transitions. Genetically, these transitions involve a series of poorly understood genetic alterations. These include loss of TSGs in several chromosomal regions, hypermutability, and alterations of housekeeping genes such as the MMR genes. ${ }^{5-8}$ Analysis of this multistep tumorigenesis in melanoma has revealed several important points. First, the genetic changes at the level of $\mathrm{BN}$ are still largely unknown. Second, the earliest genetic changes appear to involve mutations of the melanocytes of the MDN. These changes are complex and involve allelic loss, microsatellite instability (MSI), and alterations of TSGs, MMR proteins, oncogenes, and some growth factors. Third, some of these genetic alterations are shared between MDN and CMM, suggesting that some MDNs represent intermediate steps or sequential phases in CMM tumorigenesis. Finally, with the acquisition of more genetic changes, radial and vertical growth phases of melanoma develop. ${ }^{3}$

Abbreviations: $\mathrm{BN}$, common acquired melanocytic naevus; CMM, primary melanoma; hMSH, human MutS homologue; HNPCC, human non-polyposis colorectal cancer; LOH, loss of heterozygosity; MDN, melanocytic dysplastic naevi; MMR, mismatch repair; MSI, microsatellite instability; MSI- $\mathrm{H}$, high microsatellite instability; MSI-L, low microsatellite instability; RGP, radial growth phase; TSG, tumour suppressor gene; VGP, vertical growth phase 
CDNA MICROARRAY AND MELANOCYTIC LESIONS

DNA microarrays are microscopic grids of DNA, which can be used to examine gene expression within a specific cell. Using this technology, it is possible to characterise genes expressed in normal and neoplastic tissues. In the melanocytic system, the recent application of this technology revealed the upregulation of several genes in CMM, such as cyclin D1, cdc2 related protein kinase, c-Myc binding protein, early growth response protein 1 , and pleiotrophin, whereas only a few genes were slightly overexpressed in naevi. ${ }^{9}{ }^{10}$ Moreover, using a panel of antibodies for antigens involved in the cell cycle and apoptosis, melanoma antigens, transcription factors, DNA mismatch repair proteins, etc, expression profiles distinguishing specific melanoma progression stages could be identified. Most of the detected alterations in these studies were linked to the control of the Gl/S transition of the cell cycle. ${ }^{11}$ Thus, these DNA microarrays seem to have promising investigative, diagnostic, and prognostic ramifications. In this sense, expression profiling in melanocytic lesions may separate these tumours into distinct subsets and relate these subsets to definitive biological stages in the course of melanocytic transformation. ${ }^{12}{ }^{13}$

\section{LOSS OF HETEROZYGOSITY AND TSGs}

TSGs regulate normal cellular behaviour and differentiation. The release of tumour cells from growth regulatory effects accompanies the evolution of neoplasms and their subsequent progression to more malignant phenotypes. This concept is based on the following notions: (1) all cells are potentially cancerous, but this malignant potential is held in check if the cell has functioning TSGs; (2) the presence of two copies of these TSGs provides a safety backup. ${ }^{14}$ When a single copy of a TSG is lost, the surviving copy can usually maintain normal cell function. However, the surviving TSG allele may itself be destroyed by inactivating mutations. This assumption is based on the "two hit" hypothesis, suggesting that two genetic events are required for the inactivation of TSGs. ${ }^{15}$ Evidence for the presence of these suppressors can be obtained from tumour loss of heterozygosity ( $\mathrm{LOH}$ ) studies. $\mathrm{LOH}$ can be the result of chromosomal deletion, mitotic recombination, non-dysjunction, or unbalanced translocation. Only when both gene copies are lost do cells grow uncontrollably. LOH at specific regions affects TSGs and their neighbouring genes or genetic markers. These markers are usually used as flags to define the area of deletion. ${ }^{514}$

\section{MELANOMA TUMORIGENESIS BASED ON THE CHROMOSOMAL INSTABILITY AND ALLELIC LOSS PATHWAY}

Melanoma tumorigenesis involves a series of poorly understood allelic deletions at several chromosomes, including $1 \mathrm{p}$, $6 \mathrm{q}, 9 \mathrm{p}$ or $10 \mathrm{q}, 1 \mathrm{qq}$, and $17 \mathrm{q} .{ }^{16}$ One or more of the familial melanoma susceptibility genes (1p36,9p21, and 12q14) act as TSGs, and their mutation or loss probably leads to the development of melanoma. ${ }^{16-19}$ Unfolding studies indicate that the transition from a normal melanocyte to a $\mathrm{BN}$ or MDN involves the loss of genes at the $1 p$ or $9 p$ and/or $10 q$ regions. ${ }^{50-23}$ The subsequent evolution of MDN into RGPCMM involves further loss of genes on 9p, 10q, and 6q. ${ }^{54}$ Finally, the progression of VGP-CMM into metastatic melanoma involves the loss of genes at $1 \mathrm{p}, 11 \mathrm{q}, 17 \mathrm{q}$, and other undetermined chromosomes ${ }^{54-28}$ (table 1). Allelic loss analyses revealed the following: (1) certain allelic losses correlate with specific pathological stages of melanoma; (2) the presence of allelic loss in MDN and CMM suggests the inactivation of TSGs at these regions; (3) once allelic loss begins, there is a gradual increase in the amount of allelic loss, which correlates with the progression from MDN to RGP-CMM to VGP-CMM; (4) the overall accumulation of allelic losses, rather than the order in which they occur, is crucial for the development of melanomas ${ }^{524} ;(5)$ a relatively high rate of allelic loss in MDN, at 1 p36 and 9p22-21, yet similar in pattern to that of CMM, suggests the existence of a biological continuum among some MDNs and radial and vertical growth phase melanomas ${ }^{1} ;(6)$ allelic loss rates at 1p36 were low in MDN when the frequencies of allelic loss at 1p36 and 9p22 were compared in CMM and MDN, suggesting that although $\mathrm{lp}$ is involved in MDN, it plays a less important role than it does in melanomas; and (7) the absence of allelic loss at the $1 \mathrm{p}$ region in $\mathrm{BN}$ suggests that the $\mathrm{CMM} / \mathrm{MDN}$ loci may not be important in the pathogenesis of $\mathrm{BN}$ or that $\mathrm{LOH}$ may not be the mechanism of inactivation of these loci in these lesions ${ }^{5}$ (table 1; figs 1 and 2).

\section{"Unfolding studies indicate that the transition from a normal melanocyte to a benign naevus or melanocytic dysplastic naevus involves the loss of genes at the $1 p$ or $9 p$ and/or $10 \mathrm{q}$ regions"}

\section{MICROSATELLITES AND MSI}

Microsatellites are repetitive DNA sequences scattered throughout the human genome. The length of a given microsatellite repeat is unique for each individual and fixed in all the different tissues of that individual. The $(C A)_{n}$ repeat represents the most common class of microsatellite. ${ }^{43}$ The variation in microsatellite pattern length between a tumour and its matching normal tissue is referred to as microsatellite instability (MSI), and tumours with such a change are labelled as having MSI. ${ }^{43}$ Although this instability is localised to the microsatellite repeats, MSI is indicative of defective DNA repair mechanisms, and these have a general negative impact on the fidelity of replication. This negative effect may result in an increased rate of mutation in both oncogenes and TSGs, thereby leading to tumorigenesis. Moreover, MSI can generate mutations in the mutator genes. These genes have a crucial role in that they encode proteins involved in the repair of mismatched nucleotides. Furthermore, some cancer related genes, such as transforming growth factor $\beta$, have microsatellite repeats in their coding regions. In this regard, these simple repeats are the subjects for mutator specific slippage mutations. These mutations can affect cancer related genes and therefore promote tumorigenesis. ${ }^{30}$ According to the level of instability, tumours with MSI are categorised into two groups: MSI-H and MSI-L (for high and low instability, respectively). Tumours with $>30 \%$ instability of tested markers (MSI-H pattern), such as human non-polyposis colorectal cancers (HNPCCs), usually have mutations in some MMR genes. ${ }^{30}$

\section{MELANOMA TUMORIGENESIS BASED ON THE MSI PATHWAY}

In 1994, Walker and his colleagues found MSI in several melanoma cell lines, with one showing instability at several microsatellite loci. ${ }^{31}$ These findings raised the notion that a "mutator pathway", similar to that involved in HNPCC, might be implicated both in the development and progression of melanoma. Several studies have found MSI in the entire spectrum of melanocytic lesions-BN, MDN, RGP-CMM, VGP-CMM, and metastatic melanomas. ${ }^{72} 29$ 32-40 These studies revealed the following: (1) most of the melanocytic lesions had localised instability (MSI-L pattern), (2) only a small fraction of CMMs has widespread instability (MSI-H pattern), (3) only those lesions with the MSI-H pattern have MMR protein alterations and/or gene mutations, (4) MSI at dinucleotide repeats increases with melanoma progression, 
Table 1 Summary of the genetic changes in melanocytic skin lesions

\begin{tabular}{|c|c|c|c|c|c|}
\hline Aspects & $\mathrm{BN}$ & MDN & RGP-CMM & VGP-CMM & Refs \\
\hline \multicolumn{6}{|l|}{$\mathrm{LOH}$ at } \\
\hline $\begin{array}{l}\text { 1p32-36 region } \\
\text { Involved loci }\end{array}$ & $\begin{array}{l}\text { Absent } \\
-\end{array}$ & $\begin{array}{l}\text { Present (+) } \\
\text { D1S243, D1S214, } \\
\text { D1S228 }\end{array}$ & $\begin{array}{l}\text { Present }(++) \\
\text { DIS228 }\end{array}$ & $\begin{array}{l}\text { Present (+++) } \\
\text { D1S243, D1S214, } \\
\text { D1S228 }\end{array}$ & $5,16,25$ \\
\hline $5 q 21$ & - & - & - & Present $(+)$ & 18 \\
\hline Involved loci & - & - & - & D5S592 & \\
\hline $\begin{array}{l}\text { 9p22-24 } \\
\text { Involved loci }\end{array}$ & $\begin{array}{l}\text { Present (+) } \\
\text { D9S171, IFNA, D9S270 }\end{array}$ & $\begin{array}{l}\text { Present (++) } \\
\text { D9S171, D9S169, } \\
\text { D9S126 }\end{array}$ & $\begin{array}{l}\text { Present }(+++1) \\
\text { IFNA, D9S169 }\end{array}$ & $\begin{array}{l}\text { Present (++++) } \\
\text { D9S171, D9S169 }\end{array}$ & $5,20-26$ \\
\hline $10 q 22-24$ & - & - & - & Present $(+)$ & 19,27 \\
\hline Involved loci & - & - & - & DIOS520 & \\
\hline $11 q 22$ & - & - & - & & 29 \\
\hline Involved loci & - & - & - & $\begin{array}{l}\text { Present (+) } \\
\text { D11S29 }\end{array}$ & \\
\hline \multicolumn{6}{|l|}{ MSI } \\
\hline Prevalence & Present (+) & Present $(++)$ & Present $(+++)$ & Present $(++++)$ & $29-42$ \\
\hline Pattern & MSI-L & MSI-L & MSI-L & MSI-L and MSI-H & \\
\hline MMR protein expression & Present & Reduced & Reduced & Reduced & $\begin{array}{l}6,43 \\
8,44,45\end{array}$ \\
\hline MMR gene mutations & - & - & Rarely present & Rarely present & \\
\hline
\end{tabular}

BN, benign naevi; LOH, loss of heterozygosity; MDN, melanocytic dysplastic naevi; MMR, mismatch repair protien expression; MSI, microsatellite instability; MSI$\mathrm{H}$, high microsatellite instability; MSI-L, low microsatellite instability; PP, percentage of positive cells RGP-CMM, radial growth phase melanoma; VGP-CMM, vertical growth phase melanoma.

and (5) the expansion of triplet repeats can occur in melanocytic tumours (table 1; figs 1 and 2).

Moreover, these studies raise several ideas. First, MSI is involved in the pathogenesis of melanocytic lesions. This proposition is supported by three lines of evidence, namely: (a) MSI evolves during the benign stage of these lesions, (b) MSI persists throughout all the stages of melanoma tumorigenesis, and (c) MSI increases gradually with the transition from $\mathrm{BN}$ to MDN, to RGP-CMM, to VGP-CMM. Second, the lack of correlation between allelic loss and MSI in these lesions suggests that MSI may represent an

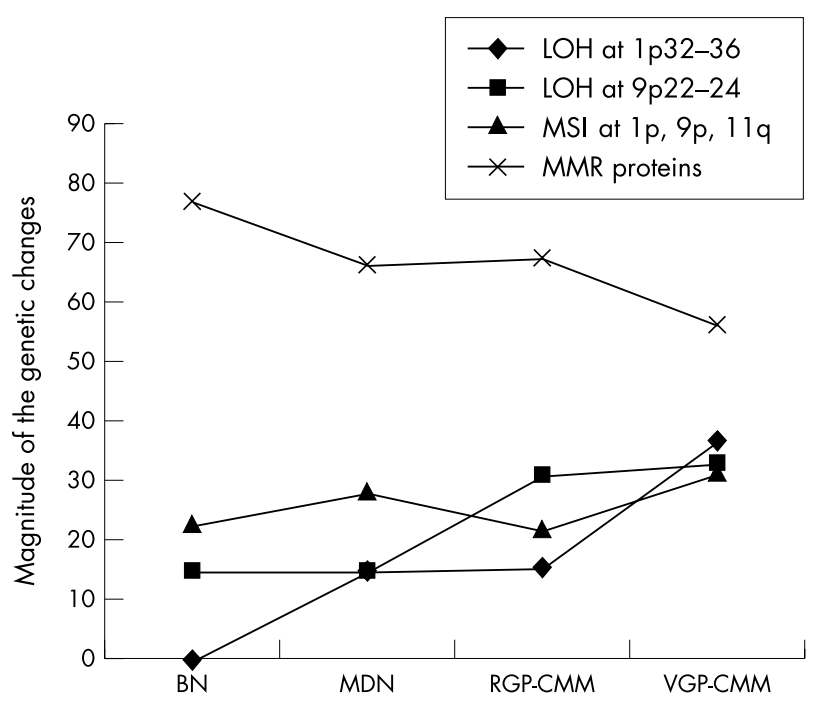

Figure 1 Genetic changes (MSI, LOH, and MMR protein alterations) during melanoma tumorigeneis. The genetic changes (increased frequency of $\mathrm{LOH}$ and $\mathrm{MSI}$ and reduced expression of MMR proteins) gradually accumulate with the transition from $B N$ to $M D N$ to RGP-CMM to VGP-CMM. Allelic loss was seen only at 9p21-22 in BN. MMR protein expression in $\mathrm{BN}$ was similar to that seen in the normal skin. $\mathrm{LOH}$ and MSI were most common at the BN stage. MMR protein alterations and gene mutations were most common in MDN and RGP-CMM melanomas, respectively. $B N$, benign naevi; $\mathrm{LOH}$, loss of heterozygosity; $M D N$, melanocytic dysplastic naevi; MMR, mismatch repair protein expression; MSI, microsatellite instability; RGP-CMM, radial growth phase melanoma; VGP-CMM, vertical growth phase melanoma. alternative and distinct pathway of tumour cell evolution. Finally, the gradual increase in the degree of MSI with the transition from $\mathrm{BN}$ to $\mathrm{MDN}$ to CMM suggests that this instability may be sequentially induced during malignant evolution, and therefore contributes to the progression of a subset of melanocytic tumours. ${ }^{78242932-42}$

We propose four different possibilities to explain the MSI-L pattern in most melanocytic lesions. First, variable expression of MMR genes with weakly penetrant mutations and an attenuated phenotype. ${ }^{46}$ A similar finding has been seen in yeast, where inactivation of human Muts homologue 3 (hMSH3) results in a less severe phenotype than does inactivation of hMSH2. ${ }^{46}$ Moreover, several studies found variability in the expression patterns of the mutator phenotype in extracts of tumour cell lines. ${ }^{47}$ Second, the inherent instability of the microsatellite loci as a part of their intrinsic properties. Third, the inactivation of MMR genes other than those encountered in HNPCC, such as hMSH6. This MMR gene member encodes the hMSH2 related protein that binds to DNA containing G/T mismatches ${ }^{48}$ Cells defective in hMSH6 showed the MSI-L pattern. Finally, increased rates of spontaneous mutations in these lesions may be responsible for this MSI-L pattern. Further investigations are required to clarify these possibilities. ${ }^{6}$

\section{"Microsatellite instability in melanocytic tumours shares three similarities with that seen in human non-polyposis colorectal cancer"}

The two MSI banding patterns seen in melanocytic lesions are similar to those described in HNPCC and familial ovarian carcinomas. ${ }^{7}$ The MSI-1 pattern, entailing the presence of additional bands, may result from division of the sequence within one of the original alleles, rather than multiplication of an existing microsatellite sequence. ${ }^{79}$ The total allelic amount and extra bands in tumour DNA have been found to be equal to the original alleles in the DNA of corresponding normal tissue. ${ }^{49}$ The partial band shifting pattern (MSI-2, limited) may be explained by the migration of CA repeat bands, as a result of instability of those repeats at one or both alleles. This change was not found to be associated with a substantial loss of DNA from the tumour tissue. ${ }^{49}$ A total band shifting pattern (MSI-2) has been reported in gliomas and sporadic ovarian carcinomas, and reflects more extensive 


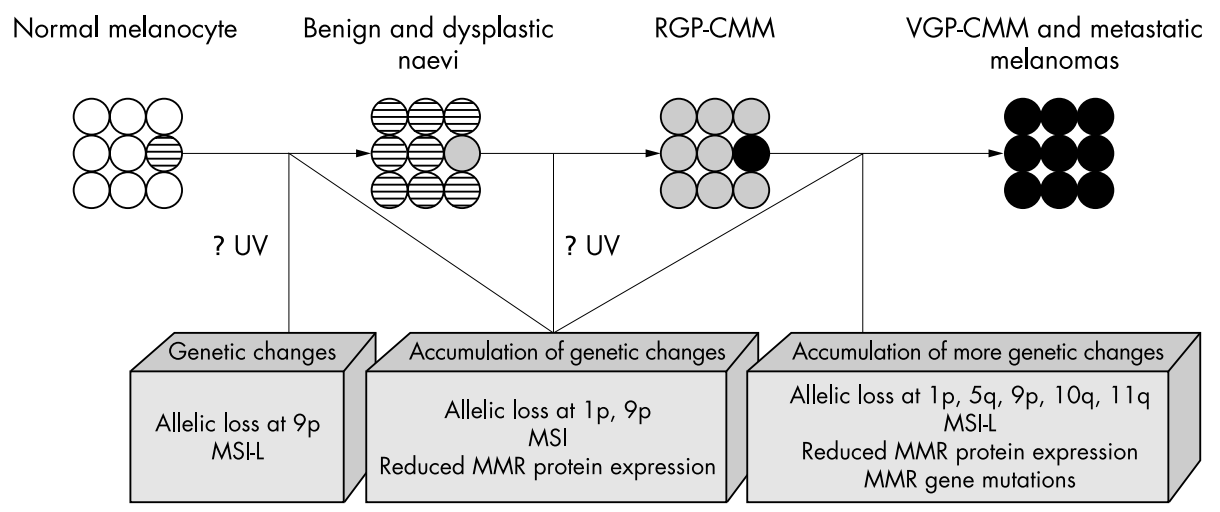

Figure 2 Melanoma tumorigenesis model. Monoclonal expansion from a polyclonal normal melanocyte is initiated by early genetic events, including $\mathrm{LOH}$ and MSI. Such expansion results in the development of BN and MDN. These expansions progess to RGP-CMM by accumulation of additional genetic events including LOH, MSI, and MMR defects. The stages of VGP-CMM and metastatic melanoma are reached after the accumulation of more crucial genetic events. Moreover, other genetic changes, such as activation of certain oncogenes, in addition to some environmental factors, such as ultraviolet (UV) exposure, may be involved. BN, benign naevi; LOH, loss of heterozygosity; MDN, melanocytic dysplastic naevi; MMR, mismatch repair protein expression; MSI, microsatellite instability; MSI-L, low microsatellite instability; RGP-CMM, radial growth phase melanoma; VGP-CMM, vertical growth phase melanoma.

changes than those associated with a partial band shift. ${ }^{50}$ MSI total band shifting may result from a higher degree of DNA instability and possibly higher mutation rates compared with partial band shifting. ${ }^{7}$

MSI in melanocytic tumours shares three similarities with that seen in HNPCC (the MSI paradigm), namely: (1) MSI begins during the first lesional step-at the BN stage, (2) it persists throughout the continuum of lesional stages, and (3) there is a sequential increase of instability with progression across this spectrum. However, MSI in melanocytic tumours differs from that seen in HNPCC in three ways, namely: (1) instability is localised rather then widespread in most of these lesions, (2) no instability is seen for mononucleotide microsatellite repeats, (3) MMR gene mutations are rare and may or may not be associated with altered protein synthesis. $^{7242932-40}$ Taken as a whole, we feel that the recent findings of MMR mutations in CMM argue against the old idea that these lesions lacking MMR gene defects. ${ }^{8}$

\section{THE MISMATCH REPAIR SYSTEM}

The MMR system identifies and repairs errors during DNA replication, and its components include the hMLH1, hMSH2, hMSH6, and human postmeiotic segregation 1 and 2 genes. ${ }^{51}$ Several studies have reported the presence of MMR protein and gene alterations in melanocytic lesions. These studies revealed several findings. First, the expression of MMR proteins in these lesions gradually decreases during the transition from BN to MDN to CMM (table 1; figs 1 and 2). Second, the concentrations of these proteins in MDN were intermediate between those of BN and VGP-CMM and close to those of RGP-CMM. ${ }^{324}$ Third, MMR gene mutations are usually associated with high level instability and are occasionally reported in melanoma cell lines. These observations further suggest that the high frequency MSI phenotype is dependent on a defective mismatch repair system. ${ }^{44}$ Fourth, in CMM, some MMR genes (hMSH2) may be regulated by p53 rather than by proliferative activity in melanocytes. ${ }^{45}$ These observations raise several ideas. First, the presence of MMR gene defects in CMM with MSI-H supports the hypothesis that in melanoma, similarly to epithelial tumours, only the high frequency MSI phenotype is strictly dependent on a defective mismatch repair system. ${ }^{45}$ Second, the absence of mutations at mononucleotide tracts indicates that, in malignant melanomas, MSI is not associated with the microsatellite mutator phenotype characteristic of mismatch repair deficient tumours. Based on these analyses, it is conceivable that if these mutations occur in the correct temporal and spatial clusters, they can contribute to the evolution of melanomas.

\section{MELANOMA TUMORIGENESIS MODEL}

Taken together, these experimental data support the notion that melanoma tumorigenesis is a multistep process requiring the sequential accumulation of a series of genetic alterations that culminate in cellular transformation. During this process, the transformed naevus cells must, in principle, progress through several molecular alterations before they can generate a clinically detectable melanoma in skin tissue. The analysis of the sequential lesional steps revealed the preferential appearance of some genetic changes at certain stages. In vivo, these transformed naevus cells must acquire many characteristics, such as growth autonomy, a capacity to evade immune surveillance processes, and the capacity to invade the surrounding tissues. These complex processes require alterations of fundamental cell processes such as: (1) accumulating mutations; (2) inactivation of TSGs, such as pl6, manifested as LOH; and (3) developing defects in housekeeping genes such as MMR genes. Defects in the MMR genes may be reflected by alterations in microsatellite repeats-that is, MSI. With the development of one or more of these alterations, only those premalignant subclones of naevus cells able to overcome the opposing selection pressure will subsequently develop into malignant ones (survival of the fittest). Those cells that survive such DNA damage probably undergo more mutations. Clonal expansion of these mutated cells may subsequently promote their progression into more aggressive stages of cancer.

\section{CONCLUSIONS}

When genetic and MMR protein alterations were compared among the different melanocytic lesions, it was evident that the extent of allelic loss, MSI, and MMR protein downregulation gradually increased in parallel with the transitions from $\mathrm{BN}$ to MDN to RGP-CMM, and finally to VGP-CMM. These data support the Clark model for melanoma tumorigenesis, and the notion that some MDN are histogenetically related and/or represent transitional steps in melanoma tumorigenesis. These observations also lend strong support to the sequential accumulation hypothesis of tumorigenesis. Over the past decade, a significant and increasing amount of experimental work has accrued, strongly supporting this 


\section{Take home messages}

- Melanoma develops as a series of lesions: common acquired melanocytic naevus to melanocytic dysplastic naevi (MDN) to radial growth phase of primary melanoma to vertical growth phase of primary melanoma and finally metastatic melanoma

- The extent of tumour suppressor gene loss, microsatellite instability, and mismatch repair protein downregulation gradually increase in parallel with this progression

- This supports the Clark model for melanoma tumor igenesis, and the notion that some MDNs are histogenetically related and/or represent transitional steps in melanoma tumorigenesis

- These observations also lend strong support to the sequential accumulation hypothesis of tumorigenesis

hypothesis. For instance, the evolution of colorectal carcinomas from benign adenomas offers some of the most compelling evidence for this theory. ${ }^{52}$ In addition, the pioneering tumorigenesis animal models of Henry Pitot also demonstrated the stepwise manner of this process. ${ }^{4}$ Overall, we believe that a unifying scheme, capable of describing tumorigenesis in a wide spectrum of spontaneous clinical cancers, will emerge. The observations and pathways presented here represent one small step towards that goal.

\section{REFERENCES}

1 Clark WH Jr, Elder DE, Guerry DT, et al. A study of tumor progression: the precursor lesions of superficial spreading and nodular melanoma. Hum Pathol 1984; 15:1147-65.

2 Ackerman AB. Malignant melanoma: a unifying concept. Hum Pathol 1980;11:591-5.

3 Hussein MR, Wood GS. Molecular aspects of melanocytic dysplastic nevi J Mol Diagn 2002;4:71-80.

4 Pitot HC. Animal models of neoplastic development. Dev Biol Basel 2001;106: 53-7; discussion 7-9, 143-60.

5 Hussein MR, Roggero E, Tuthill RJ, et al. Identification of novel deletion loci at 1 p36 and 9p22-21 in melanocytic dysplastic nevi and cutaneous malignant melanomas. Arch Dermatol 2003;139:816-17.

6 Hussein MR, Roggero E, Sudilovsky EC, et al. Alterations of mismatch repair protein expression in benign melanocytic nevi, melanocytic dysplastic nevi, and cutaneous malignant melanomas. Am J Dermatopathol 2001;23:308-14

7 Hussein MR, Sun M, Tuthill RJ, et al. Comprehensive analysis of 112 melanocytic skin lesions demonstrates microsatellite instability in melanomas and dysplastic nevi, but not in benign nevi. J Cutan Pathol 2001;28:343-50.

8 Hussein MR, Wood GS. hMLH1 and hMSH2 gene mutations are present in radial growth-phase cutaneous malignant melanoma cell lines and can be induced further by ultraviolet-B irradiation. Exp Dermatol 2003;12:872-5.

9 Seykora JT, Jih D, Elenitsas R, et al. Gene expression profiling of melanocytic lesions. Am J Dermatopathol 2003;25:6-11.

10 Pollock PM, Trent JM. The genetics of cutaneous melanoma. Clin Lab Med 2000;20:667-90.

11 Alonso SR, Ortiz P, Pollan M, et al. Progression in cutaneous malignant melanoma is associated with distinct expression profiles: a tissue microarraybased study. Am J Pathol 2004; 164:193-203.

12 Ladanyi M, Chan WC, Triche TJ, et al. Expression profiling of human tumors: the end of surgical pathology? J Mol Diagn 2001;3:92-7.

13 Dooley TP, Curto EV, Davis RL, et al. DNA microarrays and likelihood ratio bioinformatic methods: discovery of human melanocyte biomarkers. Pigment Cell Res 2003;16:245-53.

14 Marshall CJ. Tumor suppressor genes. Cell 1991;64:313-26.

15 Knudson AG Jr. Mutation and cancer: statistical study of retinoblastoma. Proc Natl Acad Sci U S A 1971:68:820-3.

16 Walker JG, Palmer JM, Walter MK. A genetic model of melanoma tumorigenesis based on allelic losses. Genes Chromosomes Cancer 1995; 12:134-41

17 Goldstein AM, Dracopoli NC, Ho EC, et al. Further evidence for a locus for cutaneous malignant melanoma-dysplastic nevus (CMM/DN) on chromosome $1 \mathrm{p}$, and evidence for genetic heterogeneity. Am J Hum Genet 1993;52:537-50.

18 Rao UN, Jones MW, Finkelstein SD. Genotypic analysis of primary and metastatic cutaneous melanoma. Cancer Genet Cytogenet 2003;140:37-44.
19 Reifenberger J, Wolter M, Bostrom J, et al. Allelic losses on chromosome arm $10 \mathrm{~g}$ and mutation of the PTEN (MMAC1) tumour suppressor gene in primary and metastatic malignant melanomas. Virchows Arch 2000;436:487-93.

20 Lee JY, Dong SM, Shin MS, et al. Genetic alterations of pl6INK4a and p53 genes in sporadic dysplastic nevus. Biochem Biophys Res Commun 1997; 237:667-72.

21 Park WS, Vortmeyer AO, Pack S, et al. Allelic deletion at chromosome 9 p21 (p16) and 17p13(p53) in microdissected sporadic dysplastic nevus. Hum Pathol 1998;29:127-30

22 Boni R, Zhuang Z, Albuquerque A, et al. Loss of heterozygosity detected on $1 \mathrm{p}$ and $9 q$ in microdissected atypical nevi [letter]. Arch Dermatol 1998; 134:882-3.

23 Boni R, Matt D, Voetmeyer A, et al. Chromosomal allele loss in primary cutaneous melanoma is heterogeneous and correlates with proliferation. $J$ Invest Dermatol 1998;110:215-17.

24 Hussein MR, Sun M, Roggero E, et al. Loss of heterozygosity, microsatellite instability, and mismatch repair protein alterations in the radial growth phase of cutaneous malignant melanomas. Mol Carcinog 2002;34:35-44.

25 Isshiki K, Elder DE, Guerry D, et al. Chromosome 10 allelic loss in malignant melanoma. Genes Chromosomes Cancer 1993;8:178-84.

26 Isshiki K, Seng BA, Elder DE, et al. Chromosome 9 deletion in sporadic and familial melanomas in vivo. Oncogene 1994;9:1649-53.

27 Herbst RA, Weiss J, Ehnis A, et al. Loss of heterozygosity for 10q22-10qter in malignant melanoma progression. Cancer Res 1994;54:3111-14.

28 Healy E, Belgaid C, Takata M, et al. Prognostic significance of allelic losses in primary melanoma. Oncogene 1998;16:2213-18.

29 Tomlinson IP, Beck NE, Bodmer WF. Allele loss on chromosome 11 q and microsatellite instability in malignant melanoma. Eur J Cancer 1996;32A: 1797-802

30 Hussein MR, Wood GS. Microsatellite instability in human melanocytic skin tumors: an incidental finding or a pathogenetic mechanism? J Cutan Pathol 2002;29:1-4.

31 Walker GJ, Palmer JM, Walters MK, et al. Simple tandem repeat allelic deletions confirm the preferential loss of distal chromosome $6 q$ in melanoma. Int J Cancer 1994;58:203-6.

32 Birindelli S, Tragni G, Bartoli C, et al. Detection of microsatellite alterations in the spectrum of melanocytic nevi in patients with or without individual or family history of melanoma. Int J Cancer 2000;86:255-61

33 Indsto JO, Cachia AR, Kefford RF, et al. X inactivation, DNA deletion, and microsatellite instability in common acquired melanocytic nevi. Clin Cancer Res 2001;7:4054-9.

34 Kroiss MM, Vogt TM, Schlegel J, et al. Microsatellite instability in malignant melanomas. Acta Derm Venereol 2001;81:242-5.

35 Massi D, Sardi I, Urso C, et al. Microsatellite analysis in cutaneous malignant melanoma. Melanoma Res 2002;12:577-84.

36 Palmieri G, Ascierto PA, Cossu A, et al. Assessment of genetic instability in melanocytic skin lesions through microsatellite analysis of benign naevi, dysplastic naevi, and primary melanomas and their metastases. Melanoma Res 2003;13:167-70.

37 Richetta A, Silipo V, Calvieri S, et al. Microsatellite instability in primary and metastatic melanoma. J Invest Dermatol 1997; 109:1 19-20.

38 Richetta A, Ottini L, Falchetti $M$, et al. Instability at sequence repeats in melanocytic tumours. Melanoma Res 2001;11:283-9.

39 Rubben A, Bogdan I, Grussendorf-Conen El, et al. Loss of heterozygosity and microsatellite instability in acquired melanocytic nevi: towards a molecular definition of the dysplastic nevus. Recent Results Cancer Res 2002;160:100-10

40 Talwalkar VR, Scheiner M, Hedges LK, et al. Microsatellite instability in malignant melanoma. Cancer Genet Cytogenet 1998;104:111-14.

41 Peris K, Keller G, Chimenti S, et al. Microsatellite instability and loss of heterozygosity in melanoma. J Invest Dermatol 1995;105:625-8.

42 Quinn AG, Healy E, Rehman I, et al. Microsatellite instability in human nonmelanoma and melanoma skin cancer. J Invest Dermatol 1995;104:309-12.

43 Hussein MR, Wood GS. Building bridges in cancer: mismatch repair and microsatellite instability. Am J Dermatopathol 2002;24:76-81

44 Alvino E, Marra G, Pagani E, et al. High-frequency microsatellite instability is associated with defective DNA mismatch repair in human melanoma. J Invest Dermatol 2002;1 18:79-86.

45 Rass K, Gutwein P, Welter C, et al. DNA mismatch repair enzyme hMSH2 in malignant melanoma: increased immunoreactivity as compared to acquired melanocytic nevi and strong mRNA expression in melanoma cell lines. Histochem J 2001:33:459-67.

46 New L, Liu K, Crouse GF. The yeast gene MSH3 defines a new class of eukaryotic MutS homologues. Mol Gen Genet 1993;239:97-108.

47 Drummond JT, Li GM, Longley MJ, et al. Isolation of an hMSH2-p160 heterodimer that restores DNA mismatch repair to tumor cells [see comments]. Science 1995;268:1909-12.

48 Palombo F, Gallinari P, laccarino I, et al. GTBP, a 160-kilodalton protein essential for mismatch-binding activity in human cells [see comments]. Science 1995;268:1912-14.

49 Arzimanoglou II, Lallas T, Osborne M, et al. Microsatellite instability differences between familial and sporadic ovarian cancers. Carcinogenesis 1996; 17:1799-804

50 Dams E, Van de Kelft EJ, Martin JJ, et al. Instability of microsatellites in human gliomas. Cancer Res 1995:55:1547-9.

51 Hussein MR, Wood GS. Microsatellite instability and its relevance to cutaneous tumorigenesis. J Cutan Pathol 2002;29:257-67.

52 Fearon ER, Vogelstein B. A genetic model for colorectal tumorigenesis. Cell 1990;61:759-67 